Ryszard Biernat

Tarnów, Poland

\title{
Quelques aspects de la spiritualité conjugale selon Jean-Marie Lustiger
}

\author{
Marital Spirituality according to Jean-Marie Lustiger
}

\section{Abstract}

In his pastoral teaching Jean-Marie Lustiger repeatedly explores the subject of marital spirituality. His teaching emerged as a response to young people's difficulty in understanding the nature of marriage as a result of being immersed in the mentality of the world. In this article the author explores two important aspects of Cardinal Lustiger's teaching on the spirituality of marriage: marriage is presented as a vocation and there is an emphasis on the correct understanding of marital love. In the context of the decline of religious practices in Western Europe, the article contains some of Jean-Marie Lustiger's thoughts on the sacrament of marriage as well as his role in contributing to a deeper understanding of matrimony.

\section{Keywords}

Jean-Marie Lustiger, marital spirituality, marital vocation, marital love, sacrament of marriage.

\section{Vocation matrimoniale}

La thèse proclamant le mariage comme une vocation n'est pas une figure stylistique. Le mariage n'est pas uniquement un état, même si chaque personne est naturellement créée pour se marier. Mais il faut le comprendre comme une 


\section{4

réponse à l'appel de Dieu pour devenir un don de soi-même $e^{1}$ et dans ce sens-là, il peut être considéré comme une vocation. Jean-Paul II a souligné, que le mariage devient une vocation chrétienne uniquement quand il reflète l'amour du Christ pour son Eglise ${ }^{2}$.

Jean-Marie Lustiger voyait dans le mariage et dans la vie consacrée une richesse multiple pour la foi catholique et constituant deux vocations données à l'Eglise. Par la vocation conjugale Dieu appelle des hommes et des femmes à vivre en ce monde, par la puissance de l'Esprit Saint, la fécondité humaine. C'est la vocation par laquelle Dieu les appelle, dans les tristesses comme dans les joies, dans les déchirements comme dans les espérances, dans les fécondités comme dans les stérilités de leur foyer, de leur travail et de leur vie, à être ceux à qui le monde est remis pour que se manifeste la puissance du Créateur qui traverse la condition de l'homme, brisée par le péché et où s'engendre déjà l'espérance d'une nouvelle création. La vocation conjugale dans la richesse de la grâce de Dieu dispense ses incroyables profondeurs et sa beauté . $^{2}$

Le bienheureux pape, en soulignant, que la Révélation chrétienne connaît deux façons spécifiques de réaliser la vocation à l'amour de la personne humaine, dans son intégrité: le mariage et la virginitét, a présenté le mariage comme une voie classique à la sainteté. Il est donc logique de constater que le mariage doit subir les mêmes mécanismes que les adeptes de la vie consacrée pour atteindre la perfection, y compris le chemin de purification. Si des conjoints ne rencontrent ni des épreuves ni des expériences sur le chemin de leur vie conjugale, cela peut signifier que leur amour conjugal ne s'est pas élevé au-delà de médiocrité . JeanMarie Lustiger constatait que la vie familiale est souvent marquée par tant de peine, d'obscurité, de souffrance. Il savait que certains époux à cause de cela doutaient, désespéraient d'eux-mêmes. Il y a des époux qui perdent conscience et espérance - conscience de ce qu'ils sont et espérance de la mission qu'ils ont reçue. Certains époux pouvaient être meurtris par la vie sociale et par les conditions économiques. Mais il enseignait que Dieu leur donne une espérance inouïe: de cette fragile cellule familiale Dieu apporte le signe et le sacrement de

\footnotetext{
${ }^{1}$ Voir: Y. Semen, Duchowość matżeńska wedtug Jana Pawła II, Poznań 2011, p. 21-22.

${ }^{2}$ « Il matrimonio corrisponde alla vocazione dei cristiani solo quando rispecchia l'amore che Cristo-Sposo dona alla Chiesa sua Sposa ». Voir: Jean-Paul II, audience, dans: http://www. vatican.va/holy_father/john_paul_ii/audiences/1982/documents/hf_jp-ii_aud_19820818_it.html. 24.04.2012, 21h39.

${ }^{3}$ Voir: J.-M. Lustiger, Femmes consacrées dans la vie religieuse, « Présence et Dialogue » (1981) n 304, p. 11.

${ }^{4}$ Voir: FC 11.

${ }^{5}$ Voir: Y. Semen, Duchowość matzeńska wedtug Jana Pawła II, op. cit., p. 70.
} 
sa présence parmi les hommes. De ce fragile amour sponsal de l'homme et de la femme, Dieu fait le signe de son amour pour l'humanité sauvée par le Christ ${ }^{6}$.

Jean-Paul II, en enseignant que le mariage, comme tout sacrement, est un mémorial, une actualisation et une prophétie de l'événement du salut, soulignait que l'Esprit, que répand le Seigneur, donne aux époux un cœur nouveau et rend l'homme et la femme capables de s'aimer, comme le Christ nous a aimés?. JeanMarie Lustiger présentait son enseignement sur le mariage en suivant cette idée. Il disait que le couple chrétien transfiguré par la grâce du sacrement de mariage est appelé à devenir le signe de l'amour unique et indéfectible du Christ pour l'Eglise ${ }^{8}$. Le sacrement du mariage révèle le lien indestructible qui unit l'homme à Dieu pour la vie et dans l'amour et il configure l'amour des époux à l'amour irrévocable du Christ pour son Eglise ${ }^{9}$. Jean-Marie Lustiger savait que la foi des jeunes qui se préparaient au mariage était fort incertaine. Ces jeunes espèraient, quand même, rendre moins fragile leur union grâce au sacrement de mariage et c'est pour cette raison-là qu'ils décidaient de se marier à l'église. Le cardinal de Paris montrait que le mariage devant Dieu n'est pas une assurance-fidélité à garantie illimitée. Il encouragait les jeunes, en leur disant, que, s'il y a plus de stabilité dans un mariage célébré à l'Eglise, c'est parce que, de ce fait, le mari et la femme ont décidé de vivre «autrement» l'histoire de leur couple et qu'ils en prennent les moyens, par la grâce de Dieu. Le sacrement de mariage prend en compte non seulement tout ce qui a précédé sa célébration à l'église, mais bien davantage, tout ce qui va le suivre. Il transforme l'histoire de deux vies en les unissant sous le regard de Dieu pour que les deux époux répondent à leur vocation $^{10}$. Il n'est que le porche d'entrée pour l'histoire future d'un homme et d'une femme dont l'union est semblable à l'amour du Christ pour son Eglise ${ }^{11}$. L'histoire commune des époux ne relève pas seulement de la construction sociale d'un couple. Elle demande à chacun des deux époux d'être solidaire de l'autre dans le mystère de la charité. Selon Jean-Marie Lustiger l'amour conjugal ne

${ }^{6}$ Voir: J.-M. Lustiger, L'Eglise et la famille, dans : $9^{\text {ème }}$ Congrès International de la famille. "La fécondité de l'amour ». Les actes du congrès, Paris 1987, édition Fayard, p. 391-392.

${ }^{7}$ Voir: FC 13.

${ }^{8}$ J.-M. Lustiger, Et Dieu vit que cela était bon (7). Un projet pour la vie, « Paris Notre Dame» (1992) n $n^{\circ} 446$, p. 8.

9 J.-M. Lustiger, Et Dieu vit que cela était bon (9). L'institution sociale du mariage, " Paris Notre Dame» (1992) no 448, p. 10.

${ }^{10}$ J.-M. Lustiger, Et Dieu vit que cela était bon (10). Un sacrement, pas une assurance tous risques, «Paris Notre Dame» (1992) n 449 , p. 8.

${ }^{11}$ Voir: J.-M. Lustiger, Et Dieu vit que cela était bon (11). Le lien de la charité, « Paris Notre Dame» $(1992) n^{\circ} 450$, p. 8. 
se définit pas seulement par une bienveillance, une gentillesse, une amabilité, une politesse et une délicatesse des sentiments, mais c'est une charité, c'est-àdire un don suprême de sa vie à l'image du Christ. Le sacrement de mariage est éminemment le sacrement de la charité. Il expliquait que le mot conjoint exprime la nature du sacrement de mariage. Le lien conjugal peut être compris comme le lien de la charité que l'Esprit Saint met entre un homme et une femme selon la parole de saint Paul: «Revêtez l'amour: c'est le lien parfait» $(\mathrm{Col} 3,14)^{12}$.

Le mariage est un état dans lequel les époux doivent accepter différentes limites. En étant une école de vie il est aussi une école de limites. Des limites du corps du conjoint, puisque le corps n'est jamais parfait. Des limites découlant du caractère du conjoint ainsi que de ses expériences, de ses blessures et de son éducation. Il est donc nécessaire d'accepter toutes ses limites et il est encore mieux de les aimer ${ }^{13}$.

En étant convaincu que le mariage est un devoir et une mission, Jean-Marie Lustiger enseignait, que Dieu demande à des hommes et des femmes faibles, vivant selon la chair et le sang, pécheurs pardonnés, d'être le signe de la vie selon l'Esprit. Dieu demande aux époux d'être dans leur amour et par la force de l'Esprit Saint, pour ceux qui n'osent plus croire, le signe de son amour pour l'homme. Il alertait les jeunes, qui ont reçu la grâce de s'engager dans le projet qui les entraîne au-delà du visible et du mesurable, qu'ils doivent aussi veiller aux conditions nécessaires pour avancer sur le chemin long et exigeant de l'amour unique et fidèle. Ils devraient le réaliser non seulement en évitant la souffrance d'un échec, le drame d'une rupture, mais aussi en apprenant à servir le bonheur de l'autre plutôt que de le faire souffrir ${ }^{14}$. L'amour doit s'enraciner en profondeur pour être plus fort que les épreuves de l'affectivité ou les oscillations des sentiments. Le cardinal de Paris a constaté que les moeurs actuelles incitent un homme et une femme qui décident de se marier à faire auparavant l'apprentissage de la vie en commun, mais il alertait les jeunes sur leurs convictions que le mariage à l'essai ou la cohabitation peut apprendre à vivre ensemble pour éviter toutes les difficultés ultérieures est une illusion ${ }^{15}$. A cette lumière le projet chrétien du mariage n'apparaît pas comme un fardeau impossible à porter, mais comme une espérance que Dieu donne à réaliser, alors même que les conditions sociales lui

${ }^{12}$ Voir: J.-M. Lustiger, Et Dieu vit que cela était bon (11). Le lien de la charité, « Paris Notre Dame» (1992) n $n^{\circ} 450$, p. 8.

${ }^{13}$ Voir: Y. Semen, Duchowość matżeńska wedtug Jana Pawła II, op. cit., p. 27.

${ }^{14}$ J.-M. Lustiger, Et Dieu vit que cela était bon (7). Un projet pour la vie, « Paris Notre Dame » (1992) n $\mathrm{n}^{\circ} 446$, p. 8.

${ }^{15}$ J.-M. Lustiger, Et Dieu vit que cela était bon (8). Si vous aimez seulement..., « Paris Notre Dame» (1992) n 447, p. 8. 
sont contraires ${ }^{16}$. Parmi des causes qui provoquent les ruptures de vie conjugales, le Cardinal de Paris évoquait un faux romantisme sentimental qui fait perdre à beaucoup le sens de la réalité pour courir après une chimère. L'amour conjugal est une mission. On n'accomplit pas une mission en rêvant, mais en avançant, en travaillant et enfin on y trouve sa joie, même dans la souffrance, d'accomplir cette mission ${ }^{17}$. Le sacrement de mariage transfigure l'union de l'homme et de la femme, pour que les époux soient prêts non seulement à s'entraider dans les conditions ordinaires de l'existence, mais aussi et surtout à travailler l'un pour l'autre, l'un par l'autre, à la vocation divine de l'un et de l'autre. Le sacrement de mariage fonde l'appel à la sainteté des époux qui est une vocation ${ }^{18}$.

\section{Amour conjugal}

Jean-Paul II, en se penchant sur l'amour conjugal, sur la sexualité et sur la sainteté de la vie matrimoniale, enseignait que tous deux, l'homme et la femme, s'éloignant de la concupiscence, trouvent la dimension de la liberté du don, unie à la féminité et à la masculinité, dans la vraie signification nuptiale et sponsale du corps. Sur cette voie la vie conjugale devient en un certain sens liturgique ${ }^{19}$.

Jean-Marie Lustiger constatait souvent que beaucoup de gens ne savent plus aimer et qu'il y a tant de gens perdus, qui ne sont aimés de personne, et que l'homme et la femme ne font plus une famille. Il disait que les gens parlent beaucoup de l'amour, mais ignorent ce qu'est l'amour. Ils veulent saisir ce qui leur plaît, en appellant cela l'amour ${ }^{20}$. L'amour n'est pas un sentiment fait de désir, d'affectivité qui ne se commande pas. La conception de l'amour comme sentiment amoureux avec sa fragilité et sa fugacité est chimérique, elle n'exprime ni le réalisme ni la profondeur de l'existence humaine ${ }^{21}$. Jean-Marie Lustiger

${ }^{16}$ Voir: J.-M. Lustiger, Et Dieu vit que cela était bon (6). L'amour, don d'une personne à une personne, «Paris Notre Dame» (1992) n 445, p. 8.

${ }^{17}$ Voir: J.-M. Lustiger, Et Dieu vit que cela était bon (15). Contre la rupture, un travail de chaque jour, «Paris Notre Dame» (1993) n 459, p. 8.

${ }^{18}$ Voir : J.-M. Lustiger, La bienheureuse espérance (9). Le sacrement du mariage, quelle espérance?, «Paris Notre Dame » (1988) nº 224, p. 10.

19 Voir: Jean-Paul II, audience du 4 juillet 1984, dans: http://www.vatican.va/holy_father/ john_paul_ii/audiences/index_fr.htm, mercredi 23 mai 2012, 16h30.

${ }^{20}$ Voir: J.-M. Lustiger, Nous avons à mettre le Coeur du Christ au centre de ce monde sans coeur, « France catholique » (1986) n 2066, p. 3.

${ }^{21}$ J.-M. Lustiger, Et Dieu vit que cela était bon (7). Un projet pour la vie, « Paris Notre Dame» (1992) n n $^{\circ} 46$, p. 8. 
dans la parole de Jésus: « Si vous aimez seulement ceux qui vous aiment, quelle récompense en aurez-vous? Les païens n'en font-ils pas autant? Moi, je vous dis, aimez vos ennemis $\rangle\rangle^{22}$, la nature même de l'amour, que ce soit l'amour conjugal ou familial, l'amour des plus proches ou des plus éloignés. Il ne peut se reconnaître seulement à la joie d'un accord affectif, ni se réduire au désir de l'homme et de la femme. L'amour implique non seulement, désir, enthousiasme, sympathie, reconnaissance mutuelle, mais aussi engagement de la liberté qui va de l'oubli de soi à l'amour de l'autre et de l'amour de l'autre à l'oubli de soi-même. Jusqu'à trouver sa joie dans le don fait et dans le don reçu; jusqu'à préférer le bonheur de l'autre à son propre bonheur et la paix de l'autre à sa propre paix ${ }^{23}$. Mari et femme ne se possèdent jamais l'un l'autre. Ils ne font que se rejoindre et s'unir en respectant ce qui les constitue l'un et l'autre, c'est-à-dire leur liberté qui les rend capables d'aimer Dieu par dessus tout ${ }^{24}$.

Jean-Marie Lustiger soulignait que la question de l'amour conjugal demande une nouvelle réflexion, car il y a un basculement qui s'est produit au sein des sociétés occidentales dans la manière de juger les comportements sexuels. Beaucoup de gens accordent à tous les comportements une même valeur. Ce qui reduit la façon de vivre l'amour et la sexualité. Selon cette mentalité tout ce qui est possible est légitime. On peut faire n'importe quoi dans la limite du consentement de chacun jusqu'à la rupture, qui peut être d'avance prévue. Ce comportement ne reconnaît pas d'autres obligations, d'autres sens à la sexualité que ce qu'impliquent les limites de l'accord des partenaires ${ }^{25}$.

Le cardinal de Paris essayait de porter la lumière de la Parole de Dieu sur les problèmes de la vie conjugale. Le constat sociologique et statistique ne suffit pas à fonder un jugement moral. La théologie chrétienne, exprimant l'originalité de la création de l'homme, dit que l'espèce humaine est composée de «personnes» dont l'identité, la nature même, est manifestée par la relation à Dieu, Père, Fils, Esprit, trois personnes divines. C'est parce qu'elle est assumée dans la relation personnelle de l'homme et de la femme, et dans leur relation à Dieu, que la sexualité devient humaine. L'empreinte divine de la création marque, transfigure toute la réalité biologique sexuée de l'homme, son intelligence, sa capacité de progrès, ses passions. La sexualité est humanisée dans la mesure où elle est

${ }^{22}$ Mt 5,46.

${ }^{23}$ J.-M. Lustiger, Et Dieu vit que cela était bon (8). Si vous aimez seulement..., « Paris Notre Dame» (1992) no 447, p. 8.

${ }^{24}$ Voir: J.-M. Lustiger, Et Dieu vit que cela était bon (13). Plus loin, plus grand que l'amour, «Paris Notre Dame» (1993) n 457, p. 8.

${ }^{25}$ Voir: J.-M. Lustiger, Et Dieu vit que cela était bon (1). Le temps de l'incertitude morale, «Paris Notre Dame» (1992) no 440, p. 8. 
assumée, comme toute notre existence, par la liberté ${ }^{26}$, car le désir, dans la mesure où il n'est pas assumé et géré par la liberté, enchaîne en esclavage celui qui s'y abandonne. Par l'érotisation de la liberté, la sexualité entraîne la liberté qui se conforme alors aux objets du désir. L'homme devient ce qu'il désire. Le désir luimême ne s'accomplit humainement que si l'homme est capable de le subordonner à sa liberté et à sa volonté de demeurer libre. La liberté est au service de l'amour.

La fidélité entre les époux suppose un amour tellement fort qu'il est capable d'assumer les contradictions du désir, un amour capable aussi de soumettre les pulsions instinctives du désir à un choix irrévocable de la liberté ${ }^{27}$. Jean-Marie Lustiger enseignait, que pour atteindre l'équilibre véritablement humain de la vie sociale et personnelle, il faut travailler à l'intégration de la sexualité dans la croissance de chaque personne et de sa capacité de don. Il faut refuser de nous représenter la fécondité conjugale à la manière du pullulement de certaines espèces animales. Il s'agit de la fécondité de l'amour qui est le propre de l'homme, c'est-à-dire le don de personne à personne, ouverture radicale à l'autre, autrement dit, au mystère de Dieu ${ }^{28}$.

Dans la réflexion sur l'amour conjugal il est nécessaire de se référer au point où se fonde tout l'édifice humain, c'est-à-dire à la dimension spirituelle de l'homme, à sa liberté. Le Cardinal de Paris était conscient que cela rencontre des obstacles dans la société contemporaine, puisque le cours des moeurs tend à nier cette dimension proprement spirituelle, éthique, du comportement sexuel, pour le réduire à des pratiques à peine ludiques ou sensuelles, sans valeur morale ni spirituelle. L'intégration de la sexualité et de l'affectivité fait partie du don des personnes l'une à l'autre. Lorsque les hommes entrent en relation les uns avec les autres, ils ne devraient pas avoir seulement des comportements instinctifs réglés à la manière des autres espèces animales. Les relations humaines sont avant tout des relations de personnes à personnes, créées à l'image de Dieu ${ }^{29}$. La vie du couple n'est pas à l'abri d'erreurs, d'accidents, du péché. L'amour qui lie des conjoints trouve sa source et sa force dans l'amour de Dieu pour sa créature,

${ }^{26}$ Voir: J.-M. Lustiger, Et Dieu vit que cela était bon (4). «A son image... homme et femme Il les créa », « Paris Notre Dame» (1992) n 443, p. 8.

${ }^{27}$ Voir: J.-M. Lustiger, Et Dieu vit que cela était bon (5). Erotiser la liberté ou libérer le désir ?, «Paris Notre Dame» (1992) nº 444, p. 8.

${ }^{28}$ Voir: J.-M. Lustiger, Et Dieu vit que cela était bon (13). Plus loin, plus grand que l'amour, «Paris Notre Dame» (1993) n 457, p. 8.

${ }^{29}$ Voir: J.-M. Lustiger, Et Dieu vit que cela était bon (6). L'amour, don d'une personne à une personne, «Paris Notre Dame» (1992) n 445, p. 8. 
dans le don qu'il nous fait de son Fils ${ }^{30}$. Il est le don de soi jusqu'à l'oubli de soi, pour l'autre. Il trouve sa réalisation la plus haute, parfaite, dans le Christ. L'idéal de l'amour conjugal trouve son origine dans l'amour indestructible qui est fondée sur la Révélation de Dieu et sur la fidélité absolue de son amour pour l'homme ${ }^{31}$.

Jean-Marie Lustiger, en citant des statistiques des années quatre-vingts du $\mathrm{XX}$-ème siècle en France qui annonçaient qu'un tiers des mariages risquait de se terminer par un divorce dans les trois ans, constatait que dans ce contexte les jeunes trouvaient dans l'amour conjugal une utopie et une chimère. Il enseignait que l'amour vrai passe par la croix $^{32}$. L'amour conjugal se construit par l'acceptation aimante et reconnaissante de l'autre différent et non par sa dévoration $^{33}$. Si un couple qui connaît les pires désillusions, si le mari et la femme ne se comprennent plus et se déchirent, si au fil du temps, ils s'éloignent l'un de l'autre et les caractères se durcissent, il reste des blessures difficiles à pardonner. Mais si l'un et l'autre se tiennent ensemble devant la croix du Christ, ils découvriront dans cette souffrance, dont ils ne voient que l'absurdité, une logique d'amour et d'oubli de soi qui leur permettra de surmonter ressentiment et amertume, de voir la haine qui pourrait se substituer à la tendresse ${ }^{34}$. Cette situation oblige à poser une question relative au pardon, qui pour des chrétiens est tout d'abord le pardon reçu de Dieu. Il n'est pas un soulagement de la culpabilité, ni un coup d'éponge qui effacerait le passé, mais il est surtout l'expérience de la résurrection des morts. Ce n'est que le pardon reçu du Pére, qui rétablit les hommes brisés dans leurs intégrités et leurs rend la vie, en les mettant debout avec le Christ, qui permet de pardonner les uns aux autres et de faire renaître l'amour ${ }^{35}$.

Les chrétiens sont appelés à chercher le centre de gravité de leur conscience dans le Christ et dans l'Eglise qui suit le Christ. Jean-Marie Lustiger savait que

${ }^{30}$ J.-M. Lustiger, Et Dieu vit que cela était bon (7). Un projet pour la vie, « Paris Notre Dame » (1992) n n $^{\circ} 46$, p. 8.

${ }^{31}$ J.-M. Lustiger, Et Dieu vit que cela était bon (9). L'institution sociale du mariage, «Paris Notre Dame » (1992) n 448, p. 10.

${ }^{32}$ Voir: J.-M. Lustiger, La bienheureuse espérance (9). Le sacrement du mariage, quelle espérance?, «Paris Notre Dame » (1988) n² 224, p. 10.

${ }^{33}$ Voir: J.-M. Lustiger, Et Dieu vit que cela était bon (11). Le lien de la charité, « Paris Notre Dame» (1992) no 450, p. 8.

${ }^{34}$ Voir: J.-M. Lustiger, Et Dieu vit que cela était bon (17). Du conflit familial au salut du monde, «Paris Notre Dame» (1993) n 461, p. 8.

${ }^{35}$ Voir: J.-M. Lustiger, La bienheureuse espérance (3). Devant l'échec apparent de l'amour, quelle espérance ?, « Paris Notre Dame» (1988) n² 218, p. 6. 
pour comprendre l'homme sauvé, il fallait se mettre dans les pas du Sauveur ${ }^{36}$ et il encourageait les hommes, baptisés dans un monde froid, à témoigner du feu de l'Evangile ${ }^{37}$. Il était convaincu, que le mystère d'un couple qui réussit chrétiennement consiste à découvrir les réalités de l'existence, quelles qu'elles soient, dans la lumière et la grâce sans cesse donnée au sacrement de mariage ${ }^{38}$.

\section{Bibliography}

Yves Semen, Duchowość matżeńska wedlug Jana Pawła II, Poznań 2011.

Jean-Marie Lustiger, Femmes consacrées dans la vie religieuse, «Présence et Dialogue » (1981) nº 304, p. 11-12.

Jean-Marie Lustiger, Nous avons à mettre le Coeur du Christ au centre de ce monde sans coeur, «France catholique » (1986) nº 2066, p. 3-4.

Jean-Marie Lustiger, L'Eglise et la famille, dans : $9^{\text {eme }}$ Congrès International de la famille. «La fécondité de l'amour ». Les actes du congrès, Paris 1987, édition Fayard, p. 389394.

Jean-Marie Lustiger, La bienheureuse espérance (3). Devant l'échec apparent de l'amour, quelle espérance?, «Paris Notre Dame » (1988) n 218, p. 6.

Jean-Marie Lustiger, La bienheureuse espérance (9). Le sacrement du mariage, quelle espérance ?, «Paris Notre Dame » (1988) nº 224, p. 10.

Jean-Marie Lustiger, Et Dieu vit que cela était bon (1). Le temps de l'incertitude morale, «Paris Notre Dame» (1992) no 440, p. 8.

Jean-Marie Lustiger, Et Dieu vit que cela était bon (2). Le poids des moeurs, «Paris Notre Dame» (1992) nº 441, p. 8.

Jean-Marie Lustiger, Et Dieu vit que cela était bon (4). «A son image... homme et femme Il les créa », «Paris Notre Dame» (1992) n 443, p. 8.

Jean-Marie Lustiger, Et Dieu vit que cela était bon (5). Erotiser la liberté ou libérer le désir ?, « Paris Notre Dame» (1992) nº 444, p. 8.

Jean-Marie Lustiger, Et Dieu vit que cela était bon (6). L'amour, don d'une personne à une personne, «Paris Notre Dame» (1992) n n 445, p. 8.

Jean-Marie Lustiger, Et Dieu vit que cela était bon (7). Un projet pour la vie, "Paris Notre Dame» (1992) nº 446, p. 8.

\footnotetext{
${ }^{36}$ Voir: J.-M. Lustiger, Et Dieu vit que cela était bon (1). Le temps de l'incertitude morale, « Paris Notre Dame» (1992) n 440, p. 8.

${ }^{37}$ Voir: J.-M. Lustiger, Et Dieu vit que cela était bon (2). Le poids des moeurs, « Paris Notre Dame» (1992) no 441, p. 8.

${ }^{38}$ Voir: J.-M. Lustiger, Et Dieu vit que cela était bon (11). Le lien de la charité, « Paris Notre Dame» (1992) no 450, p. 8.
} 
Jean-Marie Lustiger, Et Dieu vit que cela était bon (8). Si vous aimez seulement..., «Paris Notre Dame » (1992) no 447, p. 8.

Jean-Marie Lustiger, Et Dieu vit que cela était bon (9). L'institution sociale du mariage, «Paris Notre Dame» (1992) n 448, p. 10.

Jean-Marie Lustiger, Et Dieu vit que cela était bon (10). Un sacrement, pas une assurance tous risques, « Paris Notre Dame» (1992) n 449, p. 8.

Jean-Marie Lustiger, Et Dieu vit que cela était bon (11). Le lien de la charité, «Paris Notre Dame » (1992) no 450, p. 8.

Jean-Marie Lustiger, Et Dieu vit que cela était bon (13). Plus loin, plus grand que l'amour, «Paris Notre Dame » (1993) n 457, p. 8.

Jean-Marie Lustiger, Et Dieu vit que cela était bon (15). Contre la rupture, un travail de chaque jour, « Paris Notre Dame » (1993) no 459, p. 8.

Jean-Marie Lustiger, Et Dieu vit que cela était bon (17). Du conflit familial au salut du monde, « Paris Notre Dame » (1993) no 461, p. 8. 